\title{
Efeitos da fotobiomodulação e exercícios na dor e força muscular na osteoartrose de joelho: Uma revisão sistemática
}

\author{
Effects of photobiomodulation and exercises on pain and muscle strength in knee osteoarthrosis: A \\ systematic review \\ Efectos de la fotobiomodulación y ejercicios sobre el dolor y la fuerza muscular en la osteoartrosis \\ de rodilla: Una revisión sistemática
}

\section{Resumo}

Introdução: A osteoartose consiste em uma doença degenerativa que pode ou não estar associada a processos inflamatórios, um dos recursos terapêuticos possíveis para o tratamento dessa enfermidade é a fotobiomodulação laser. Objetivos: Este estudo tem como objetivo, analisar por meio de uma revisão sistemática de literatura, a eficácia da fotobiomodulação e exercícios na da dor e força muscular na osteoartrose de joelho. Metodologia: Foi realizado um levantamento bibliográfico nas bases de dados Medline, PubMed e Scielo, utilizando como descritores: osteoartrose, fotobiomodulação, fisioterapia, terapia a laser. Foram identificados 35 artigos com ano de publicação entre 2011-2020, escritos nos idiomas português e inglês. Posteriormente, foram lidos os resumos dos artigos, eliminando-se 29 artigos que não atenderam aos critérios. Resultados: 6 artigos compuseram essa revisão, demonstraram diferentes parâmetros de laser infravermelho, e diferentes protocolos de exercícios, porém todos com bons resultados. Conclusão: conclui-se que o uso da fotobiomodulação do laser infravermelho associado ao treino de força tem grande eficácia no tratamento de pacientes com osteoartrose de joelho.

Palavras-chave: Osteoartrose; Fisioterapia; Terapia a laser.

\begin{abstract}
Introduction: Osteoartosis is a degenerative disease that may or may not be associated with inflammatory processes, one of the possible therapeutic resources for the treatment of this disease is laser photobiomodulation. Objectives: This study aims to analyze, through a systematic literature review, the effectiveness of photobiomodulation and exercises in pain and muscle strength in knee osteoarthritis. Methodology: A bibliographic survey was carried out in the Medline, PubMed and Scielo databases, using as descriptors: osteoarthritis, photobiomodulation, physiotherapy, laser therapy. 35 articles were identified with year of publication between 2011-2020, written in Portuguese and English. Subsequently, the abstracts of the articles were read, eliminating 29 articles that did not meet the criteria. Results: 6 articles comprised this review, demonstrated different infrared laser parameters, and different exercise protocols, but all with good results. Conclusion: it is concluded that the use of infrared laser photobiomodulation associated with strength training has great efficacy in the treatment of patients with knee osteoarthritis.
\end{abstract}

Keywords: Osteoarthritis; Physiotherapy; Laser therapy.

\section{Resumen}

Introducción: La osteoartosis es una enfermedad degenerativa que puede o no estar asociada a procesos inflamatorios, uno de los posibles recursos terapéuticos para el tratamiento de esta enfermedad es la fotobiomodulación con láser. Objetivos: Este estudio tiene como objetivo analizar, a través de una revisión sistemática de la literatura, la efectividad de la fotobiomodulación y los ejercicios en el dolor y la fuerza muscular en la artrosis de rodilla. Metodología: Se realizó un levantamiento bibliográfico en las bases de datos Medline, PubMed y Scielo, utilizando como descriptores: 
artrosis, fotobiomodulación, fisioterapia, terapia con láser. Se identificaron 35 artículos con año de publicación entre 2011-2020, escritos en portugués e inglés. Posteriormente, se leyeron los resúmenes de los artículos, eliminando 29 artículos que no cumplían con los criterios. Resultados: 6 artículos componían esta revisión, demostraron diferentes parámetros de láser infrarrojo, y diferentes protocolos de ejercicio, pero todos con buenos resultados. Conclusión: se concluye que el uso de fotobiomodulación con láser infrarrojo asociado al entrenamiento de fuerza tiene una gran eficacia en el tratamiento de pacientes con artrosis de rodilla.

Palabras clave: Artrosis; Fisioterapia; Terapia con láser.

\section{Introdução}

A osteoartose (OA) consiste em uma doença degenerativa que pode ou não estar associada a processos inflamatórios. Ocorre devido ao desgaste da articulação podendo estar envolvidos menisco, cartilagem ou osso subcondral. A OA pode ser classificada em primária quando sua etiologia é desconhecida, ou secundária quando associadas a processos inflamatórios (Dadalto et al, 2013).

A OA é mais incidente, em mulheres do que em homens, possui incidência mundial de 9,6\% nos homens e $18 \%$ nas mulheres com idade superior a 60 anos. Cerca de $80 \%$ possuem limitação de movimento e $25 \%$ apresentam dificuldades em realizar as atividades de vida diária. (Sardim et al, 2020). Ainda que não seja clara, alguns fatores são apontados, como hormonais, obesidade e menor volume articular nas mulheres quando comparadas com os homens. A dor é o motivo mais frequente de queixa entre os portadores da $\mathrm{AO}$, por isso os principais objetivos do tratamento são o alivio da dor, diminuição da rigidez articular melhorando a qualidade de vida (Moreira et al, 2014).

Os aspectos clínicos da OA consistem em dor, rigidez matinal, crepitações, diminuição da amplitude de movimento e consequentemente diminuição da função e qualidade de vida. No Raio x é possível observar a diminuição do espaço intraarticular, formações de osteófitos, esclerose do osso subcondral e formações císticas. (Duarte et al, 2013).

A redução da força muscular e da capacidade funcional também pode ser evidente por mudanças na rigidez do tendão patelar, visto que uma redução na rigidez do tendão afeta a taxa de desenvolvimento da força e, por conseguinte, as AVD'S. (Blum et al, 2020).

A fraqueza do quadríceps pode diminuir a capacidade de absorção de choque durante a caminhada levando a maiores sintomas, consequentemente o paciente altera o padrão de marcha com o intuito de minimizar a dor e manter a funcionalidade. Portanto esse déficit de força interfere drasticamente na sua 13 qualidade de vida. (Carlos et al, 2012)

O diagnóstico é feito através da anamnese, exame físico, e exames radiográficos onde se pode encontrar a presença de osteófitos, cistos e esclerose do osso subcondral, contudo o indicador mais evidente da OA é o estreitamento da lacuna articular (Flugsrud et al, 2010).

Abordando a osteoartrose de joelho de uma perspectiva psicossocial e não apenas pelas alterações patológicas, podemos distinguir três níveis de atenção. O primeiro nível, pode ser aplicado a todos os pacientes, os métodos devem incluir educação em saúde, com recomendações e informações sobre a mesma. O segundo nível é constituído pela reabilitação, utilização de recursos a auxiliares de marcha, assim como o tratamento farmacológico (agentes analgésicos e antiinflamatórios). Os pacientes com um alto grau de incapacidade e dor continua apesar da terapêutica instituída, deve ser considerada a indicação à consulta de ortopedia podendo ser necessário tratamento cirúrgico (terceiro nível). (Da Costa ,2015)

A fisioterapia tem como objetivo melhorar a força e a resistência muscular, melhorar a mobilidade articular, promover analgesia e consequentemente melhora na qualidade de vida (Bentes \& Bossini,2018). Além do tratamento cinesioterapêutico alguns recursos eletrotermofototerapêuticos são utilizados para analgesia e anti-inflamatórios, como a estimulação transcutânea nervosa, ultrassom, e o laser de baixa frequência. O laser de baixa frequência é uma fonte de luz monocromática que possui um efeito não térmico e estimula a reparação da cartilagem (Fukuda et al, 2011). 
A fotobiomodulação (FBM) laser vai estimular a membrana celular e suas mitocôndrias, causando uma série de eventos fisiológicos dentre eles o aumento da produção de ATP, favorece a produção de ácido araquidônico e a transformação de prostaglandina em prostaciclina, justificando sua ação antiedematosa e anti-inflamatória e promove aumento da endorfina circulante proporcionando o efeito analgésico na dor inflamatória (De Avelar et al, 2020).

O principal efeito bioelétrico da FBM laser é a manutenção do potencial de membrana celular, o que impede que os estímulos dolorosos se propaguem a centros nervosos, isso devido à eficiência da bomba de sódio e potássio ocasionada pela maior disponibilidade de ATP resultante do efeito bioquímico. O efeito analgésico desta modalidade terapêutica se deve à sua ação em diversos níveis. Localmente, ocorre redução da inflamação por meio da reabsorção de exsudatos e da eliminação de substâncias algiogênicas (Frare \& Nicolau,2008).

Este estudo tem como objetivo analisar, por meio de uma revisão sistemática de literatura, os efeitos da fotobiomodulação e exercícios na dor e força muscular em indivíduos com osteoartrose de joelho.

\section{Metodologia}

O presente estudo trata-se de uma revisão sistemática, de natureza quantitativa, realizada entre o período de fevereiro a maio de 2021, a fim de compor o presente artigo foi realizado um levantamento bibliográfico nas bases de dados Medline, PubMed e Scielo, utilizando-se como descritores: osteoartrose, fisioterapia, terapia a laser, e seus descritores em inglês “osteoarthritis", "physiotherapy", "laser therapy".

Os trabalhos selecionados e inclusos na pesquisa constituíram-se apenas de artigos originais e ensaios clínicos, a fim de ter melhor resultado e conclusão sobre o tema.

Os artigos selecionados e inclusos na pesquisa constituíram-se apenas de artigos dos últimos dez anos, na língua inglesa e portuguesa, ensaios clínicos. Serão excluídas revisões sistemáticas, estudo de caso, pesquisa com animais.

Para a seleção do material, foram necessárias 3 etapas (Figura 1). A primeira foi caracterizada pela busca do material nas bases de dados, de fevereiro/2021 a maio/2021, nas quais selecionam-se 35 artigos. A segunda etapa constitui-se da leitura de cada título e resumo para melhor conhecer e compreender o assunto, excluindo os que não tinham relevância com o tema. Após a seleção, buscaram-se os textos na íntegra que estavam disponíveis, escolhendo-se 6 trabalhos para compor esta revisão.

\subsection{Avaliação da qualidade metodológica dos artigos}

A escala usada para avaliação dos artigos foi a escala de PEDro (Tabela 1). Foi desenvolvida pela Physiotherapy Evidence Database para ser empregada em estudos experimentais e tem uma pontuação total de até 10 pontos, incluindo critérios de avaliação de validade interna e apresentação da análise estatística empregada. Ele visa quantificar a qualidade dos ensaios clínicos aleatorizados publicados, de forma a guiar os usuários sobre os aspectos meritórios de cada publicação e facilitar a identificação rápida de estudos que contenham informações suficientes para a prática profissional. A escala PEDro é composta pelos seguintes critérios: 1) especificação dos critérios de inclusão (item não pontuado); 2) alocação aleatória; 3) sigilo na alocação; 4) similaridade dos grupos na fase inicial ou basal; 5) mascaramento dos sujeitos; 6) mascaramento do terapeuta;7) mascaramento do avaliador; 8) medida de pelo menos um desfecho primário em 85\% dos sujeitos alocados; 9) análise da intenção de tratar; 10) comparação entre grupos de pelo menos um desfecho primário e 11) relato de medidas de variabilidade e estimativa dos parâmetros de pelo menos uma variável primária. 


\section{Resultados e Discussão}

Figura 1 - Fluxograma de busca dos estudos incluídos.
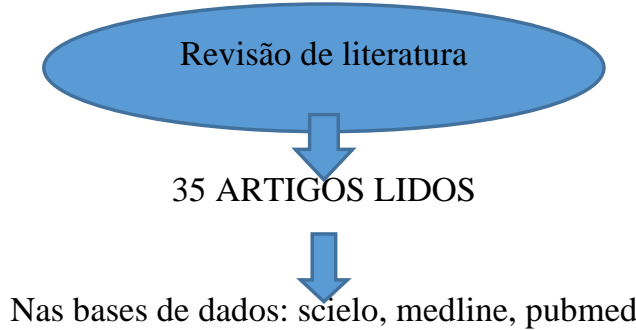

Incluídos: Artigos originais

\section{Ensaios clínicos}

Artigos na língua inglês e português

Artigos dos últimos der anne

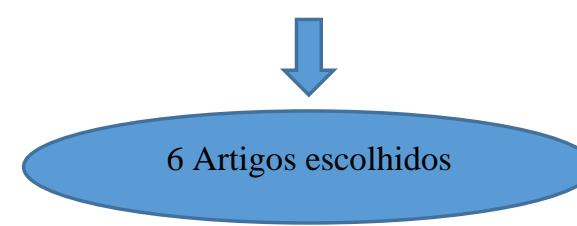

\section{Artigos escolhidos}

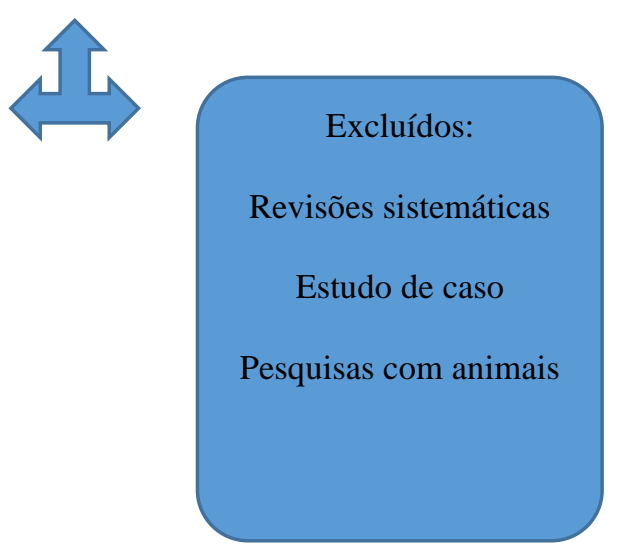

Fonte: Autores (2021). 
Tabela 1 - Classificação dos ensaios clínicos randomizados de acordo com a escala Pedro.

\begin{tabular}{|c|c|c|c|c|c|c|}
\hline Escala PEDro & $\begin{array}{l}\text { Alfredo, PP, } \\
2017\end{array}$ & SARDIM,2020 & $\begin{array}{c}\text { VASSÃO, } \\
\text { Patricia } \\
\text { Gabrielli et } \\
\text { al } 2018\end{array}$ & $\begin{array}{c}\text { VASSÃO, } \\
\text { Patricia } \\
\text { Gabrielli et } \\
\text { al. } 2018\end{array}$ & $\begin{array}{c}\text { De Paula } \\
\text { Gomes } \\
\text { CAF et } \\
\text { al,2018 }\end{array}$ & $\begin{array}{c}\text { ALFRE } \\
\text { DO PP } \\
2011\end{array}$ \\
\hline $\begin{array}{l}\text { Critérios de } \\
\text { elegibilidade }\end{array}$ & SIM & SIM & SIM & SIM & SIM & SIM \\
\hline Distribuição aleatória & 1 & 1 & 1 & 1 & 1 & 1 \\
\hline $\begin{array}{c}\text { Alocação secreta dos } \\
\text { sujeitos }\end{array}$ & 1 & 1 & 1 & 1 & 1 & 1 \\
\hline $\begin{array}{c}\text { Semelhança inicial entre } \\
\text { os grupos }\end{array}$ & 1 & $\mathbf{0}$ & 1 & 1 & 1 & 1 \\
\hline Cegamento dos sujeitos & 1 & 1 & 1 & 1 & 1 & 1 \\
\hline $\begin{array}{l}\text { Cegamento dos } \\
\text { terapeutas }\end{array}$ & 1 & 1 & 1 & 1 & 1 & 1 \\
\hline $\begin{array}{c}\text { Cegamento dos } \\
\text { avaliadores }\end{array}$ & 1 & $\mathbf{0}$ & 1 & 1 & 1 & 1 \\
\hline $\begin{array}{c}\text { Acompanhamento } \\
\text { adequado }\end{array}$ & $\mathbf{0}$ & $\mathbf{0}$ & $\mathbf{0}$ & $\mathbf{0}$ & $\mathbf{0}$ & $\mathbf{0}$ \\
\hline $\begin{array}{c}\text { Análise da intenção de } \\
\text { tratamentos }\end{array}$ & $\mathbf{0}$ & $\mathbf{0}$ & $\mathbf{0}$ & $\mathbf{0}$ & $\mathbf{0}$ & $\mathbf{0}$ \\
\hline $\begin{array}{l}\text { Comparação } \\
\text { intergrupos }\end{array}$ & 1 & 1 & 1 & 1 & 1 & 1 \\
\hline $\begin{array}{c}\text { Medidas de precisão e } \\
\text { variabilidade }\end{array}$ & 1 & $\mathbf{0}$ & 1 & 1 & 1 & 1 \\
\hline Escore total & $8 / 10$ & $5 / 10$ & 8/10 & $8 / 10$ & 9/10 & 8/10 \\
\hline
\end{tabular}

Fonte: PEDro.

$\mathrm{Na}$ avaliação da qualidade metodológica, todos os estudos apresentaram uma pontuação na escala PEDro $\geq 4$ pontos. Assim, foram classificados como de alta qualidade de acordo com os critérios de Van Peppen et al. (2004). 
Research, Society and Development, v. 10, n. 7, e2010716668, 2021

(CC BY 4.0) | ISSN 2525-3409 | DOI: http://dx.doi.org/10.33448/rsd-v10i7.16668

Tabela 2 - Características dos trabalhos originais selecionados, publicados entre 2011 a 2020.

\begin{tabular}{|c|c|c|c|c|c|c|}
\hline Autor/Ano & $\begin{array}{l}\text { Tipo de } \\
\text { estudo }\end{array}$ & Descrição da amostra & $\begin{array}{l}\text { Instrumentos } \\
\text { de coleta de } \\
\text { dados }\end{array}$ & Intervenção & $\begin{array}{c}\text { Variávei } \\
\text { s } \\
\text { avaliada } \\
\text { s }\end{array}$ & Desfechos significativos \\
\hline $\begin{array}{l}\text { SARDIM, André } \\
\text { Cabral; PRADO, } \\
\text { Rodrigo Paschoal; } \\
\text { PINFILDI, Carlos } \\
\text { Eduardo,2020 }\end{array}$ & $\begin{array}{l}\text { Estudo } \\
\text { longitudinal, } \\
\text { prospectivo e } \\
\text { simples cego } \\
\text { com } \\
\text { resultados } \\
\text { preliminares. }\end{array}$ & $\begin{array}{l}\text { Vinte indivíduos } \\
\text { selecionados foram } \\
\text { distribuídos em dois } \\
\text { grupos grupo controle } \\
\text { (GC) foi submetido à } \\
\text { aplicação de FBM } \\
\text { placebo e ao protocolo } \\
\text { de exercício enquanto o } \\
\text { grupo fotobiomodulação } \\
\text { (GF) foi submetido à } \\
\text { aplicação ativa da FBM } \\
\text { e ao protocolo de } \\
\text { exercício. }\end{array}$ & $\begin{array}{l}\text { Questionários } \\
\text { SF-36, } \\
\text { Lequesne e } \\
\text { Tinetti, álém da } \\
\text { EVA. }\end{array}$ & $\begin{array}{l}\text { Os pacientes foram } \\
\text { submetidos ao tratamento } \\
\text { durante oito semanas com } \\
\text { uma frequência de duas } \\
\text { vezes na semana por uma } \\
\text { hora por sessão. } \\
\text { Inicialmente realizavam } \\
\text { alongamento passivo } \\
\text { estático, após alongamento } \\
\text { treino de fortalecimento, ao } \\
\text { final foi aplicado a FBM em } \\
\text { dois pontos anteriormente } \\
\text { na linha articular do joelho, } \\
\text { com comprimento de onda } \\
850 \mathrm{~nm} \text { e } 670 \mathrm{~nm} \text {, potência } \\
\text { de saída } 100 \mathrm{~nm} \text { e10nm, } \\
\text { energia por ponto } 30 \mathrm{~J} \text { com } \\
\text { densidade } 4 \mathrm{~J} / \mathrm{cm} 2 \\
\text { estacionária em contato com } \\
\text { a pele. }\end{array}$ & $\begin{array}{l}\text { Dor, } \\
\text { funcional } \\
\text { idade. }\end{array}$ & $\begin{array}{l}\text { Pode-se dizer que o uso da } \\
\text { FBM, associada a } \\
\text { exercícios, apresentou melhora } \\
\text { na dor dos pacientes com } \\
\text { osteoartrite de joelho, embora } \\
\text { não tenha sido possível } \\
\text { observar diferenças } \\
\text { significativas no que dissesse } \\
\text { respeito } \\
\text { à funcionalidade. }\end{array}$ \\
\hline $\begin{array}{c}\text { Vassão, PG, de } \\
\text { Souza, MC, Silva, } \\
\text { BA et al } 2020\end{array}$ & $\begin{array}{l}\text { Ensaio clinico } \\
\text { randomizado } \\
\text { controlado } \\
\text { por placebo. }\end{array}$ & $\begin{array}{l}\text { tgf } \\
62 \text { mulheres divididas } \\
\text { em dois grupos. }\end{array}$ & $\begin{array}{l}\text { Escala numérica } \\
\text { da dor (NPRS), } \\
\text { Teste de } \\
\text { caminhada de } 6 \\
\text { minutos(6- } \\
\text { MWT), teste } \\
\text { cronometrado } \\
\text { (TUG), torque } \\
\text { isométrico } \\
\text { máximo } \\
\text { (MVIT). }\end{array}$ & 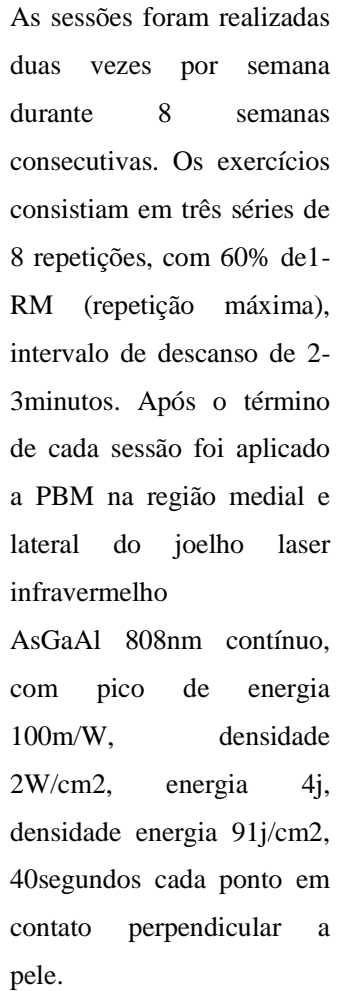 & $\begin{array}{l}\text { Dor, } \\
\text { capacida } \\
\text { de física, } \\
\text { força } \\
\text { muscular } \\
\text {. }\end{array}$ & $\begin{array}{l}\text { Os principais resultados } \\
\text { demonstraram que todas as } \\
\text { Voluntárias tratadas } \\
\text { apresentaram uma diminuição } \\
\text { significativa no } \\
\text { Nível de dor após } 8 \text { semanas de } \\
\text { intervenção em comparação com } \\
\text { as } \\
\text { Mulheres tratadas com placebo. } \\
\text { Além disso, a análise intragrupo } \\
\text { Mostrou que o TC6 e a carga do } \\
\text { MVIT apresentaram valores } \\
\text { Maiores para os voluntários } \\
\text { treinados (com ou sem PBM) na } \\
\text { reavaliação. O TUG foi maior } \\
\text { para todos os grupos tratados } \\
\text { comparando os valores pré e } \\
\text { pós-tratamento. }\end{array}$ \\
\hline
\end{tabular}


Research, Society and Development, v. 10, n. 7, e2010716668, 2021

(CC BY 4.0) | ISSN 2525-3409 | DOI: http://dx.doi.org/10.33448/rsd-v10i7.16668

\begin{tabular}{|c|c|c|c|c|c|c|}
\hline $\begin{array}{c}\text { De Paula Gomes CAF } \\
\text { et al.2018 }\end{array}$ & $\begin{array}{l}\text { Ensaio clínico } \\
\text { controlado } \\
\text { por placebo, } \\
\text { randomizado }\end{array}$ & $\begin{array}{l}59 \text { Indivíduos do sexo } \\
\text { masculino e feminino } \\
\text { com idades } \\
\text { Entre } 40-80 \text { anos } \\
\text { joelho,alocados } \\
\text { aleatoriamente a um } \\
\text { grupo submetido a } \\
\text { exercício sozinho, um } \\
\text { grupo submetido a } \\
\text { exercício } \\
\text { fototerapia ativa ou } \\
\text { grupo submetido a } \\
\text { exercícios ofototerapia } \\
\text { com placebo. }\end{array}$ & $\begin{array}{l}\text { WOMAC, } \\
\text { Numerical } \\
\text { Rating Pain } \\
\text { Scale (NRPS), } \\
\text { Lower } \\
\text { Extremity } \\
\text { Functional } \\
\text { Scale (LEFS), } \\
\text { dinamômetro } \\
\text { Portátil, Teste } \\
\text { de } \\
\text { Alcance } \\
\text { Funcional } \\
\text { (TRF). }\end{array}$ & $\begin{array}{l}\text { Foram realizados para } \\
\text { aumentar a força muscular } \\
\text { dos músculos glúteo } \\
\text { máximo e médio, } \\
\text { quadríceps com duas séries } \\
\text { de quinze repetições e } \\
\text { progrediu para três séries de } \\
\text { quinze repetições com } 70 \% \\
\text { de 1-RM. Ao final de cada } \\
\text { sessão de exercícios foi } \\
\text { aplicado a fototerapia } \\
\text { usando um laser de diodo } \\
\text { pulsado de } 905 \mathrm{~nm} \text {, potência } \\
\text { 8,5W, quatro leds, utilizado } \\
\text { sobre três quadrantes do } \\
\text { joelho. }\end{array}$ & $\begin{array}{l}\text { Dor, } \\
\text { força } \\
\text { muscular } \\
\text { equilíbrio } \\
\text { função } \\
\text { física. }\end{array}$ & $\begin{array}{l}\text { Analisando os efeitos clínicos } \\
\text { das intervenções propostas, a } \\
\text { Combinação de exercícios a } \\
\text { fototerapia ativa foi } \\
\text { significativamente mais eficaz } \\
\text { do que o exercício sozinho e } \\
\text { exercício fototerapia com } \\
\text { placebo apenas em relação ao } \\
\text { NRPS, diferença } \\
\text { Considerando a outras } \\
\text { clinicamente importante mínima. } \\
\text { Em relação às } \\
\text { comparações entre os grupos, } \\
\text { não foram encontrados } \\
\text { resultados clínicos significativos } \\
\text { para função, limiar de dor à } \\
\text { pressão, força muscular ou } \\
\text { equilíbrio }\end{array}$ \\
\hline ALFREDO PP ,2011 & $\begin{array}{l}\text { Ensaio clinico } \\
\text { randomizado. }\end{array}$ & $\begin{array}{l}46 \text { Pacientes entre } 50-75 \\
\text { anos com AO de joelho } \\
\text { foram divididos em dois } \\
\text { grupos, laser e placebo. }\end{array}$ & $\begin{array}{l}\text { Escala visual } \\
\text { analógica } \\
\text { visual, } \\
\text { Goniômetro } \\
\text { visual, } \\
\text { Dinamômetro } \\
\text { portátil, } \\
\text { Questionário } \\
\text { Lequesne, } \\
\text { TimedGeTUpan } \\
\text { dg } \\
\text { Go(TUG),Wom } \\
\text { ac. }\end{array}$ & $\begin{array}{l}\text { Foi utilizado o laser de Asga } \\
\text { comprimento de onda } \\
904 \mathrm{~nm}, \quad 700 \mathrm{hz} \text { de } \\
\text { frequência, potência } 60 \mathrm{~mW} \text {, } \\
\text { potência de pico } 20 \mathrm{~W} \text {, } \\
\text { duração de pulso } \\
4,3 \text { milessegundos } \\
\text { Feixe de } 0,5 \mathrm{~cm} 2 \text {, irradiadas } \\
\text { por cinco pontos. O } \\
\text { programa de exercícios } \\
\text { durou } 45 \text { minutos realizado } \\
\text { oito semanas cada exercício } \\
\text { teve } 3 \text { series de } 20 \\
\text { repetições com progressão } \\
\text { de carga. }\end{array}$ & $\begin{array}{l}\text { Dor, } \\
\text { força } \\
\text { muscular } \\
\text { amplitud } \\
\text { e de } \\
\text { movimen } \\
\text { to, } \\
\text { funcional } \\
\text { idade. }\end{array}$ & $\begin{array}{l}\text { No grupo laser foi observado } \\
\text { maior ganho relativo em todas as } \\
\text { avaliações. }\end{array}$ \\
\hline $\begin{array}{c}\text { Alfredo, PP, Bjordal, } \\
\text { JM, Junior, WS, } \\
\text { Lopes-Martins, R. } \\
\text { Á. B., Stausholm, } \\
\text { MB, Casarotto, } \\
\text { RA,... Joensen, J. } \\
\text { (2017). }\end{array}$ & $\begin{array}{l}\text { Estudo } \\
\text { randomizado, } \\
\text { controlado, } \\
\text { duplo-cego. }\end{array}$ & $\begin{array}{l}\text { Quarenta participantes } \\
\text { de ambos os sexos, com } \\
\text { idades entre 50-75 anos } \\
\text { com osteoartrite de } \\
\text { joelho grau 2-4 }\end{array}$ & $\begin{array}{l}\text { Dor em uma } \\
\text { escala visual } \\
\text { analógica, } \\
\text { consumo de } \\
\text { paracetamol e } \\
\text { gravidade da } \\
\text { osteoartrite } \\
\text { medida pelo } \\
\text { Índice de } \\
\text { Osteoartrite das } \\
\text { Universidades } \\
\text { Western } \\
\text { Ontario } \\
\text { McMaster } \\
\text { (WOMAC) e } \\
\text { Índice } \\
\text { Lequesne. }\end{array}$ & $\begin{array}{l}\text { O grupo LLLT recebeu } 10 \\
\text { tratamentos LLLT com laser } \\
\text { infravermelho invisível (904 } \\
\mathrm{nm}, 3 \text { Joules / ponto) } \\
\text { durante três semanas, } \\
\text { seguido por um programa de } \\
\text { exercícios de fortalecimento } \\
\text { supervisionado de oito } \\
\text { semanas. O grupo LLLT } \\
\text { placebo recebeu tratamento } \\
\text { idêntico, mas a saída do } \\
\text { laser infravermelho foi } \\
\text { desativada. }\end{array}$ & $\begin{array}{l}\text { Dor, } \\
\text { força } \\
\text { muscular } \\
\text { amplitud } \\
\text { e de } \\
\text { movimen } \\
\text { to } \\
\text { passiva. }\end{array}$ & $\begin{array}{l}\text { As melhorias imediatas de um } \\
\text { programa de tratamento que } \\
\text { consiste em LLLT mais } \\
\text { exercícios de fortalecimento } \\
\text { supervisionados parecem ser } \\
\text { mantidas por seis meses após o } \\
\text { final do tratamento }\end{array}$ \\
\hline
\end{tabular}




\begin{tabular}{|c|c|c|c|c|c|c|}
\hline $\begin{array}{l}\text { VASSÃO, Patricia } \\
\text { Gabrielli et al } 2018\end{array}$ & $\begin{array}{l}\text { Ensaio clínico } \\
\text { prospectivo, } \\
\text { controlado } \\
\text { por placebo, } \\
\text { duplo-cego e } \\
\text { randomizado. }\end{array}$ & $\begin{array}{l}35 \text { mulheres saudáveis } \\
\text { entre } 60 \text { e } 70 \text { anos de } \\
\text { idade foram divididas } \\
\text { em dois grupos: Grupo } \\
\text { Placebo }(n=13) \text { e Grupo } \\
\text { Ativo }(n=14) \text {. }\end{array}$ & $\begin{array}{l}\text { Teste 1-MR, } \\
\text { Teste de } \\
\text { caminhada de } \\
\text { seis minutos, } \\
\text { Bateria de } \\
\text { desempenho } \\
\text { físico curto } \\
\text { (SPPB), Teste } \\
\text { de Equilíbrio. }\end{array}$ & $\begin{array}{l}\text { O protocolo de exercício } \\
\text { consistiu no exercício de } \\
\text { flexão e extensão de joelhos } \\
\text { seguido da aplicação da } \\
\text { fotobiomodulação placebo } \\
\text { ou ativa, } 2 \text { vezes por semana } \\
\text { por } 8 \text { semanas consecutivas. }\end{array}$ & $\begin{array}{l}\text { Força } \\
\text { muscular } \\
\text { equilíbrio }\end{array}$ & $\begin{array}{l}\text { Aumento significativo no } \\
\text { TC6 }(\mathrm{p}=0,001) \text {, SPPB }(\mathrm{p}= \\
0,006) \text { e 1-RM }(\mathrm{p}=0,001) \\
\text { em ambos os grupos. O } \\
\text { programa de treinamento de } \\
\text { força associado a } \\
\text { fotobiomodulação ativa } \\
\text { promoveu um aumento } \\
\text { significativo relativo à } \\
\text { avaliação inicial, no índice } \\
\text { de estabilidade medial / } \\
\text { direito lateral ( } \mathrm{p}=0,007) \mathrm{e} \\
\text { diminuição no Teste de } \\
\text { Risco de Queda }(\mathrm{p}=0,005) .\end{array}$ \\
\hline
\end{tabular}

Fonte: Elaborado pelos autores (2020)

A OA é uma moléstia muito comum e se apresenta entre $44 \%$ e $70 \%$ nos indivíduos com mais de 50 anos de idade; na faixa etária acima de 75 anos, esse percentual sobe para 85\%. Além disso, corresponde uma das principais queixas da consulta médica e é responsável por um número excessivo de ausência do trabalho e aposentadorias por invalidez. É um distúrbio musculoesquelético, geralmente insidioso, progressivo e lento, que afeta tipicamente as articulações das mãos, da coluna, do quadril e do joelho. (Lima et al, 2019)

Além de produzir um estado inflamatório crônico, a doença geralmente causa muitas consequências psicossociais prejudiciais aos pacientes. A dor contínua, incapacidade funcional, limitações econômicas e efeitos colaterais das drogas terapêuticas, que a OA pode ocasionar, podem acabar reduzindo a qualidade de vida desses pacientes. (Mella et al, 2010)

A OA, causa sofrimento prolongado e leva o paciente à dependência física e perda de autonomia, a dor e suas implicações para o estado físico e mental dos pacientes com OA parecem ser componentes importantes e afetam de forma decisiva a qualidade de vida desses pacientes. (Ferreira et al, 2015.)

A dor causada pela OA, leva ao desuso gerando uma atrofia, com isso esses pacientes apresentam uma redução de 50$60 \%$ da força de quadríceps, resultando na redução da força extensora do joelho. (Rodrigues et al, 2013).

A diminuição na ativação muscular é uma possível explicação para a perda de força muscular e capacidade funcional. Um estudo anterior observou uma inibição significativa do quadríceps na OA em comparação com participantes saudáveis, o que justifica o comprometimento da força dos músculos extensores do joelho. (Blum et al, 2020).

Portanto o presente estudo teve como objetivo analisar, por meio de uma revisão sistemática de literatura, os efeitos da fotobiomodulação e exercícios na dor e força muscular em indivíduos com osteoartrose de joelho. As propostas de tratamento dos estudos presentes nesta revisão sistemática foram satisfatórias quando comparado às intervenções placebo, principalmente na melhora do quadro de dor desses pacientes.

É notório que a dor nas articulações é um dos sintomas mais evidenciado pelos pacientes com OA e está relacionada ao desgaste da cartilagem, ao processo inflamatório do tecido ligado à articulação afetada e às alterações neuromusculares. (Vassão et al, 2019).

Araújo et al (2011) sugere que a luz na faixa espectral do vermelho ao infravermelho próximo poderia contribuir na recuperação da cartilagem lesionada, atuando de forma positiva no controle da OA. Esse efeito pode ser explicado pelo estímulo à proliferação de condrócitos e pelo aumento da produção de glicosaminoglicanos e mucopolissacarídeos, componentes que garantem a integridade da cartilagem articular, induzidos pela terapia luminosa. A fotobiomodulação gera 
moléculas de oxigênio reativas que são capazes de interagir com os tecidos corporais, induzindo ou inibindo reações químicas no interior das células.

Os trabalhos inclusos na presente revisão utilizaram doses de aplicação de $3-4 \mathrm{~J} / \mathrm{cm}^{2}$. Segundo a literatura, doses excitatórias (até $8 \mathrm{~J} / \mathrm{cm}^{2}$ ) são apropriadas quando o objetivo da intervenção inclui a potencialização da ação da bomba de sódio e potássio, o estímulo à produção de ATP, o restabelecimento do potencial de membrana, o aumento do metabolismo e da proliferação celular.

Fukuda e colaboradores (2011) realizaram ensaio clínico randomizado cego e controlado para avaliar a eficácia dos efeitos da fotobiomodulação a curto prazo na melhora da dor e função em pacientes com OA de joelho. Participaram 47 voluntários de ambos os sexos que foram divididos em dois grupos (placebo e ativo). No grupo ativo, os parâmetros 30 utilizados foram: laser AsGa de $904 \mathrm{~nm}, 60 \mathrm{~mW}$ de potência média e $0,5 \mathrm{~cm}^{2}$ de área do feixe, irradiados nove pontos no joelho com energia de 3,0J por ponto. O tratamento do grupo ativo foi realizado três vezes por semana (nove sessões). Os resultados apontaram significativa melhora na dor e função no grupo ativo quando comparado ao grupo placebo.

Sabe-se que programas de exercícios diminuem a síntese de mediadores inflamatórios das articulações e também a apoptose dos condrócitos, diminuindo o nível de dor em pacientes com OA. A FBM é apta a modular o processo inflamatório por meio da estimulação celular na via respiratória mitocondrial, ocasionando benefícios fisiológicos e clínicos. (Vassão et al, 2018).

Com a diminuição do torque articular ocorre uma perda progressiva da força muscular. O quadríceps é o mais acometido, que é o responsável pela absorção de choques e pela estabilização da articulação do joelho. Essa fraqueza gera uma perda progressiva de função, facilitando o avanço da doença. (Alfredo et al, 2011)

Vários estudos apontam que o fortalecimento do quadríceps seria o ponto chave para controlar a dor, a função física e a qualidade de vida dessa população. Além disso esse fortalecimento contribui para a melhora no controle da oscilação postural, consequentemente, melhora as habilidades para a realização das tarefas funcionais. (Oliveira et al, 2012)

Vassão et al (2020) não identificaram efeitos adicionais da PBM a um programa de exercícios na dor, rigidez, capacidade funcional, força muscular e sua influência nas alterações posturais em pacientes com osteoartrose de joelho. Justificam possíveis resultados pela alta dose de energia total emitida no cluster (4J energia por ponto, 56J total por joelho) como um dos possíveis fatores para os resultados.

Em contrapartida, em outra revisão sistemática, compararam os efeitos da PBM com o placebo em indivíduos diagnosticados com OAJ e apontaram diferenças entre a terapia e o placebo, a favor da terapia, na dor em repouso, dor nas atividades, dor total, os domínios função e rigidez do WOMAC e pontuação total do WOMAC, entretanto sem diferenças na amplitude de movimento e no domínio dor do WOMAC. (Lima, 2020)

Contudo, a fotobiomodulação combinada com exercícios pode ampliar o leque de tratamento dos profissionais no mercado de trabalho, além de incentivar novas pesquisas nessas modalidades de tratamento na osteoartrose de joelho.

\section{Conclusão}

Esse estudo abre uma janela para aplicações clinicas. Através dos resultados encontrados nos artigos selecionados foi possível verificar a eficácia da analgesia causada pela fotobiomodulação do laser infravermelho, demonstrando assim efetividade na diminuição da dor e seu efeito analgésico. E quando somada a exercícios mostram grandes resultados no que diz respeito a melhora da dor e ganho de força.

Desta forma, os achados nessa revisão sistemática sugerem que o laser de baixa intensidade associado a exercícios podem trazer bons resultados no tratamento de pacientes com osteoartrose de joelho. 
No entanto, ressaltamos que é de grande valia a realização de mais estudos clínicos, utilizando a fotobiomodulação laser em conjunto com exercícios mais específicos para fortalecimento de quadríceps, visto que é o grupo muscular mais afetado.

Aos autores que tiverem interesse em pesquisar acerca do tema, foquem na dosimetria com maior eficácia para esse tipo de enfermidade. Buscando um consenso a respeito dos melhores parâmetros do laser (dose, comprimento de onda) e protocolos associados que possam levar ao objetivo desejado.

\section{Referências}

Alfredo, P. P., Bjordal, J. M., Junior, W. S., Lopes-Martins, R. Á. B., Stausholm, M. B., Casarotto, R.A., \& Joensen, J. (2017). Resultados a longo prazo de um estudo randomizado, controlado e duplo-cego da terapia a laser de baixa potência antes dos exercícios na osteoartrite do joelho: laser e exercícios na osteoartrite do joelho. Reabilitação Clínica, 32 (2), 173-8. doi: 10.1177 / 0269215517723162

do Nascimento Bentes, R., \& dos Santos Bossini, E. (2018). Efeitos da mobilização com movimento em associação ao tratamento fisioterapêutico sobre a qualidade de vida e dor na osteoartrose de joelho. Fisioterapia Brasil, 19(3)

Blum, D., Rodrigues, R., Geremia, J. M., Brenol, C. V., Vaz, M. A., \& Xavier, R. M. (2020). Propriedades do músculo quadríceps na artrite reumatóide: percepções sobre a morfologia muscular, ativação e capacidade funcional. Advances in Rheumatology, 60, 1-9.

Carlos, K. P., Belli, B. D. S., \& Alfredo, P. P. (2012). Efeito do ultrassom pulsado e do ultrassom contínuo associado a exercícios em pacientes com osteoartrite de joelho: estudo piloto. Fisioterapia e Pesquisa, 19(3), 275-281.

Dadalto, T. V., Souza, C. P. D., \& Silva, E. B. D. (2013). Eletroestimulação neuromuscular, exercícios contrarresistência, força muscular, dor e função motora em pacientes com osteoartrite primária de joelho. Fisioterapia em movimento, 26(4), 777-789.

da Costa, D. L. M. (2015). Avaliação da referenciação dos doentes com osteoartrose do joelho dos cuidados de saúde primários para a consulta de especialidade de ortopedia: Artigo de investigação médica.

de Paula Gomes, C. A., Leal-Junior, E. C., Dibai-Filho, A. V., de Oliveira, A. R., Bley, A. S., Biasotto-Gonzalez, D. A., \& de Tarso Camillo de Carvalho, P. (2018). Incorporation of photobiomodulation therapy into a therapeutic exercise program for knee osteoarthritis: A placebo-controlled, randomized, clinical trial. Lasers in surgery and medicine, 50(8), 819-828.

Duarte, V. D. S., Santos, M. L. D., Rodrigues, K. D. A., Ramires, J. B., Arêas, G. P. T., \& Borges, G. F. (2013). Exercícios físicos e osteoartrose: uma revisão sistemática. Fisioterapia em Movimento, 26(1), 193-202.

Frare, J. C., \& Nicolau, R. A. (2008). Análise clínica do efeito da fotobiomodulação laser (GaAs-904 nm) sobre a disfunção temporomandibular. Brazilian Journal of Physical Therapy, 12(1), 37-42.

Ferreira, A. H., Godoy, P. B. G., Oliveira, N. R. C. D., Diniz, R. A. S., Diniz, R. E. A. S., Padovani, R. D. C., \& Silva, R. C. B. D. (2015). Investigação da ansiedade, depressão e qualidade de vida em pacientes portadores de osteoartrite no joelho: um estudo comparativo. Revista Brasileira de Reumatologia, 55(5), 434-438.

Fukuda, V. O., Fukuda, T. Y., Guimarães, M., Shiwa, S., Lima, B. D. C. D., Martins, R. Á. B. L., \& Fucs, P. M. M. B. (2011). Eficácia a curto prazo do laser de baixa intensidade em pacientes com osteoartrite do joelho: ensaio clínico aleatório, placebo-controlado e duplo-cego. Revista Brasileira de Ortopedia, 46(5), 526-533.

Lima, D. A. D., Gonçalves, M. C. K., Grando, S. T. C., Cintra, T. L. D. L., Pinto, D. M., \& Gonçalves, R. K. (2019). Indicações da neurotomia dos nervos geniculares por radiofrequência para o tratamento da osteoartrite do joelho: uma revisão de literatura. Revista Brasileira de Ortopedia, 54(3), 233-240.

Lima, G. E. G. (2020). Efeitos da fotobiomodulação e cinesioterapia no tratamento da osteoartrite de joelho: estudo clínico, prospectivo e randomizado.

Mella, L. F. B., Bértolo, M. B., \& Dalgalarrondo, P. (2010). Sintomas depressivos em pacientes com artrite reumatoide. Brazilian Journal of Psychiatry, 32(3), $257-263$.

Moreira, M., Afonso, M., \& Araújo, P. (2014). Anti-inflamatórios não esteróides tópicos no tratamento da dor por osteoartrose do joelho-Uma revisão baseada na evidência. Revista Portuguesa de Medicina Geral e Familiar, 30(2), 102-8.

Oliveira, A. M. I. D., Peccin, M. S., Silva, K. N. G. D., Teixeira, L. E. P. D. P., \& Trevisani, V. F. M. (2012). Impacto dos exercícios na capacidade funcional e dor em pacientes com osteoartrite de joelhos: ensaio clínico randomizado. Revista Brasileira de Reumatologia, 52(6), 876-882.

Rodrigues, R. C., Pontin, J. C. B., Falcon, S. M., \& Chamlian, T. R. (2013). Avaliação do perfil, satisfação e efetividade do tratamento fisioterapêutico em grupo nos pacientes com osteoartrite de joelho. Acta fisiátrica, 20(3), 124-128.

Sardim, A. C., Prado, R. P., \& Pinfildi, C. E. (2020). Efecto de la fotobiomodulación asociada a ejercicios sobre el dolor y la funcionalidad en pacientes con osteoartritis de rodilla: un estudio piloto. Fisioterapia e Pesquisa, 27(2), 119-125.

Vassão, P. G., Toma, R. L., Antunes, H. K. M., \& Renno, A. C. M. (2018). Photobiomodulation and physical exercise on strength, balance and functionality of elderly women. Fisioterapia em Movimento, 31.

Vassão, P. G., De Souza, M. C., Silva, B. A., Junqueira, R. G., de Camargo, M. R., Dourado, V. Z., \& Renno, A. C. (2020). Fotobiomodulação por meio de um dispositivo de cluster associado a um programa de exercícios físicos no nível de dor e força muscular em mulheres de meia-idade e idosas com osteoartrite de joelho: um ensaio randomizado controlado por placebo. Lasers in medical science, 35 (1), 139-148. 